\title{
Conical-emission and shock-front dynamics in femtosecond laser-pulse filamentation
}

\author{
F. Bragheri, ${ }^{1, *}$ D. Faccio, ${ }^{2}$ A. Couairon,${ }^{3}$ A. Matijosius, ${ }^{4}$ G. Tamošauskas, ${ }^{4}$ A. Varanavičius, ${ }^{4}$ V. Degiorgio, ${ }^{1}$ A. Piskarskas,${ }^{4}$ \\ and P. Di Trapani ${ }^{4, \dagger}$ \\ ${ }^{1}$ Department of Electronics, University of Pavia, Via Ferrata 1, I-27100 Pavia, Italy \\ ${ }^{2}$ CNR-INFM, Department of Physics and Mathematics, University of Insubria, Via Valleggio 11, 22100 Como, Italy \\ ${ }^{3}$ Centre de Physique Théorique, CNRS, École Polytechnique, F-91128, Palaiseau, France \\ ${ }^{4}$ Department of Quantum Electronics, Vilnius University, Sauletekio Ave. 9, 10222 Vilnius, Lithuania
}

(Received 12 September 2006; published 16 August 2007)

\begin{abstract}
We investigate both experimentally and numerically the space-time dynamics of an ultrashort laser pulse during self-focusing and nonlinear propagation in water by means of a time-gated angular-spectrum characterization. The results identify the formation of shock fronts on both trailing and leading edges of the wave packet that are due to the formation of subluminal and superluminal group velocity intensity peaks, sustained by conical emission.
\end{abstract}

DOI: 10.1103/PhysRevA.76.025801

PACS number(s): 42.65.Tg, 41.20.Jb, 42.65.Jx, 42.65.Sf

Temporal-shock formation manifests itself as the selfsteepening of a wave packet (WP) propagating in a nonlinear material when the velocity of the peak differs from that of the wings [1]. This general phenomenon, featuring acoustics, electromagnetic microwaves, and plasmas [2], had been widely investigated in the sixties also in the optical range. For example, in the framework of a plane-wave model, a Kerr nonlinearity leading to a refraction index that increases with intensity $\left(n_{2}>0\right)$ supports a peak that travels slower than the rest of the pulse. This creates a shock on the trailing edge (a "trailing shock"), accompanied by a broadening of the blueshifted part of the spectrum [1]. Although in real settings dispersion will not allow self-steepening to form an infinite gradient, we still speak of shock-front formation when the front approaches its minimum allowed duration. Trailing shocks, outlined by wave-breaking oscillations, were later observed in optical fibers [3]. As to propagation in bulk media and so the interplay between space and time effects, Gaeta has demonstrated the link existing between shock dynamics and the spectacular supercontinuum generation (SCG) [4]. Specifically, in the investigated regime (i.e., the catastrophic collapse in a $5 \mathrm{~mm}$ sapphire sample), spacetime focusing and self-steepening were shown to create a trailing shock too, consistently with what was presented also in a later investigation on SCG [5-8]. In this scenario, the blueshifted SCG is enhanced, while the redshifted SCG is expected to be weaker. Yet in a regime of ultrashort-pulse filamentation in condensed media, where longer samples, looser focusing, and higher power are typically adopted [9], numerics and experiments have shown redshifted axial SCG even further extended than the blueshifted side $[10,11]$. Notably, evidence of self-steepening of the rising front can be seen in previous numerical works (e.g., [7,12]), although the emergence of leading shocks has never been explicitly discussed.

Recently, the spatiotemporal reshaping featuring

\footnotetext{
*francesca.bragheri@unipv.it

†Permanent address: Department of Physics and Mathematics, University of Insubria, Via Valleggio 11, 22100 Como, Italy.
}

ultrashort-pulse filamentation has been explained as the spontaneous formation of nonlinear $X$ waves [12]. This interpretation links the observed colored conical emission (CCE) to the inherent $X$-wave angular dispersion (i.e., the dependence of temporal frequencies on angles) [13]. In the context of $X$-wave modeling, axial SCG has been interpreted as the result of a phase mismatched three-wave-mixing interaction $[12,14]$. However, no connection is given to shockfront formation as the analysis has been limited to the far field. In this Brief Report we report on the evidence of the interplay between shock front and $X$-wave dynamics. The result is obtained by implementing an experimental technique capable of monitoring the angular spectrum (AS) of each temporal slice of the optical pulse while it propagates in the nonlinear medium, thus linking the features in the farfield spectrum to those in the near-field intensity profile. The method allows us to demonstrate the existence not only of trailing but also of leading shock fronts, which were never considered before.

The gated angular spectrum technique (GAS) adopted in our experiment is based on a nonlinear gating, performed by frequency mixing in a $\chi^{(2)} \mathrm{BBO}$ crystal the WP under investigation with an ultrashort optical gate [15]. The generated sum frequency (SF) signal reproduces, at shorter wavelengths, the pulse field profile within the gate. By changing the delay between pulse and gate and by recording the AS of the SF signal for each delay, we determine the temporal origin of the various (angle and frequency) components of the WP spectrum. The adopted imaging refractive optics add different delays to different frequencies so that numerical experiments have been used for an interpretation of the results. Here, we investigate CCE and shock-front dynamics of $160 \mathrm{fs}, 800 \mathrm{~nm}$ laser pulses undergoing filamentation in water. The input pulse, delivered by a Ti:sapphire laser system (Spitfire, Spectra-Physics) at $1 \mathrm{kHz}$ repetition rate, was loosely focused into the nonlinear sample. The diameter of the Gaussian beam on the entrance face of the cuvette was $100 \mu \mathrm{m}$. The propagation distance was fixed at $z=3 \mathrm{~cm}$ and the input energy at $3.3 \mu \mathrm{J}$. This corresponds to an input peak power $P \simeq 11 P_{\mathrm{cr}}$, with the critical power for self-focusing defined as $P_{\mathrm{cr}}=3.77 \lambda^{2} /\left(8 \pi n_{0} n_{2}\right)$, where $\lambda, n_{0}, n_{2}=3.2$ $\times 10^{-16} \mathrm{~cm}^{2} / \mathrm{W}$ are the vacuum wavelength and the linear 


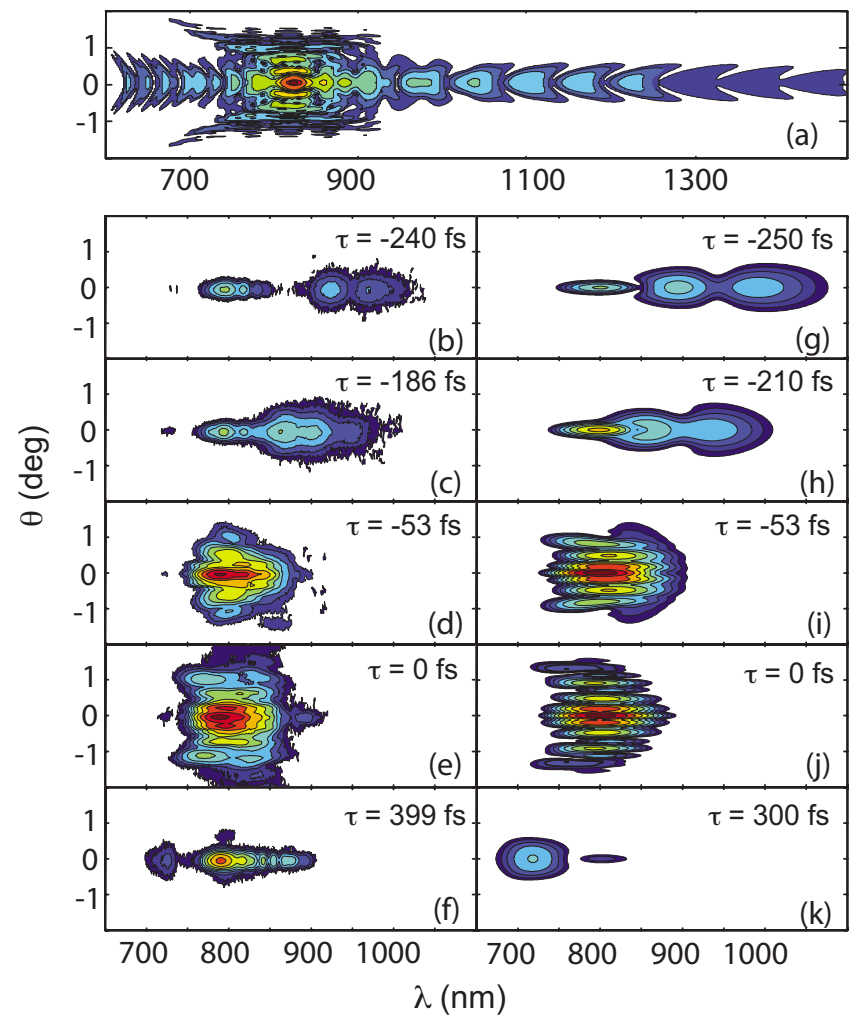

FIG. 1. (Color online) (a) Numerically calculated timeintegrated AS of a $160 \mathrm{fs} 800 \mathrm{~nm}$ Gaussian beam focused into a 3 -cm-length cuvette filled with water. [(b)-(k)] Angular Spectra of different temporal slices of the WP under investigation. Left column: experiment. Right column: numerical simulations.

and nonlinear refractive index, respectively. Figure 1(a) shows a numerically calculated spectrum (model described below and input conditions as in the experiment), which highlights the main spectral features under investigation. Blueshifted CCE is observed with relatively large angles while the redshifted CCE is much less visible and confined to smaller angles due to the vicinity of the zero-dispersion wavelength [16]. The on-axis $(\theta \sim 0)$ spectrum is dominated by the supercontinuum that exhibits new frequencies on both blue and red sides. We have observed similar spectra experimentally for a wide range of materials, including water with the same input conditions $[10,17]$. Figures $1(\mathrm{~b})-1(\mathrm{f})$ show the measured GAS for increasing delay times. The zero delay is defined with respect to the peak of a linearly propagating input Gaussian pulse. For large negative delays axial redshifted emission is very evident [Fig. 1(b)]. Moving closer to zero delay [Fig. 1(c)] the wavelength shift decreases; in other words, we observe a frequency chirp. For time slices centered around zero delay [Figs. 1(e) and 1(f)] the axial redshifted or blueshifted components disappear and are replaced by a strong off-axis generation corresponding to CCE. Finally, on the trailing part of the WP [Fig. 1(f)] we observe no CCE but again axial emission, this time blueshifted with respect to the input wavelength.

In order to underline the origin of the temporal chirp, we propose in Fig. 2(a) a different representation of the same results, by plotting the measured temporal (angular-

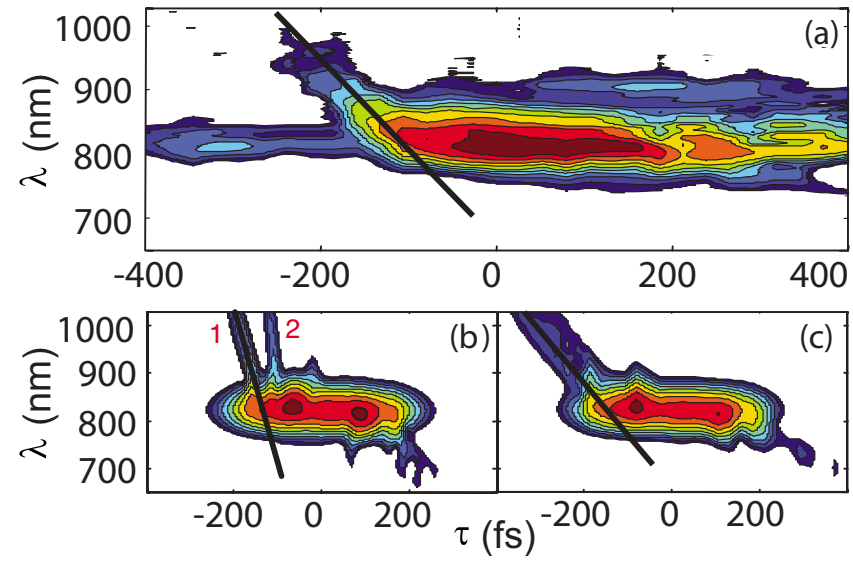

FIG. 2. (Color online) Gated angular spectrum (GAS) characterization of a $160 \mathrm{fs} 800 \mathrm{~nm}$ Gaussian beam focused into a 3-cm-long water sample: Experimental (a) and computed (b) logarithmic intensity distribution of wavelength versus delay; (c) same as (b) with the addition of $1 \mathrm{~cm}$ of linear propagation in fused silica.

integrated) spectrum as a function of delay. The figure highlights a clear linear chirp of the redshifted spectral components at negative delays (solid black line in the figure, with slope $-0.2 \mathrm{~nm} / \mathrm{fs}$ ), which have temporal duration $T_{1}$ $\simeq 110 \mathrm{fs}$ centered at $\tau \simeq-170 \mathrm{fs}$. Relying upon data presented in Fig. 1, we interpret this as the redshifted component of the axial continuum and we attribute this emission as due to a rising steep front in the temporal pulse profile. In order to estimate the (minimum) half width at half maximum duration $T_{0}$ of this front inside the medium, we should account for the chirp introduced by the two lenses (fused silica, $\sim 1 \mathrm{~cm}$ total thickness) used to image the output facet of the water sample onto the nonlinear gating crystal. By exploiting the equation $T_{1}=T_{0}\left[1+\left(z\left|\beta_{2}\right| / T_{0}^{2}\right)^{2}\right]^{1 / 2}$, where $\beta_{2}=362$ $\mathrm{fs}^{2} / \mathrm{cm}$ is the group velocity dispersion (GVD) coefficient of fused silica, we obtain $T_{0}=3$ fs, i.e., a figure compatible with a rising shock front in the leading portion of the wave packet. Finally, we note that a weak falling front in the blueshifted spectral region at positive delays $(\tau \simeq 300 \mathrm{fs})$ is noticeable in Fig. 2(a), which should be linked with the axial blueshifted continuum seen in Fig. 1.

In what follows we present the results of our numerical experiments. The model [11] is based on the nonlinear equation governing the evolution of the envelope $\mathcal{E}(r, t, z)$ of the linearly polarized pulse of central frequency $\omega_{0}$, along the propagation axis as follows:

$$
\frac{\partial \mathcal{E}}{\partial z}=\frac{i}{2 k_{0}} U^{-1} \nabla_{\perp}^{2} \mathcal{E}-i \frac{k^{\prime \prime}}{2} \frac{\partial^{2} \mathcal{E}}{\partial t^{2}}+\frac{k^{\prime \prime \prime}}{6} \frac{\partial^{3} \mathcal{E}}{\partial t^{3}}+U^{-1} N(\mathcal{E}),
$$

where $t \equiv t_{\mathrm{lab}}-z / v_{g}$ denotes the retarded time, $k(\omega)$ $\equiv n(\omega) \omega / c$ the wave number of the Fourier components, $k_{0}$ $\equiv k\left(\omega_{0}\right), v_{g} \equiv \partial \omega /\left.\partial k\right|_{\omega_{0}}$ denotes the group velocity, and $U$ $\equiv\left(1+\frac{i}{k_{0} v_{g}} \frac{\partial}{\partial t}\right)$. Equation (1) accounts for diffraction in the transverse plane, space-time focusing [4], and GVD up to third order term (the dispersive coefficients are $k^{\prime \prime}$ $\equiv \partial^{2} k /\left.\partial \omega^{2}\right|_{\omega_{0}}$ and $\left.k^{\prime \prime \prime} \equiv \partial^{3} k /\left.\partial \omega^{3}\right|_{\omega_{0}}\right)$ : 


$$
N(\mathcal{E})=i \frac{\omega_{0}}{c} n_{2} T^{2}|\mathcal{E}|^{2} \mathcal{E}-T \frac{\beta_{K}}{2}|\mathcal{E}|^{2 K-2} \mathcal{E}
$$

The nonlinear term $N(\mathcal{E})$ includes the optical Kerr effect and nonlinear losses (NLL) with order $K=5$ and cross section $\beta_{K}=1 \times 10^{-48} \mathrm{~cm}^{7} / \mathrm{W}^{4}$. The operator $T \equiv 1+\frac{i}{\omega_{0}} \frac{\partial}{\partial t}$ in front of the Kerr term is responsible for pulse self-steepening and optical shocks $[4,18]$. Due to the relatively low peak intensities reached under our experimental conditions $\left(\sim 3 \mathrm{TW} / \mathrm{cm}^{2}\right)$ and small pulse duration, self-generated plasma has been neglected (see also comments in Ref. [4]). The linear propagation in the dispersive imaging optics is also suitably accounted for. Figures $1(\mathrm{~g})-1(\mathrm{k})$ show the computed GAS slices for input pulse, nonlinear material, and imaging conditions as in our experiments. The optimum agreement with experimental results under such detailed diagnostics indicates the adequateness of the chosen model and numerical tool for the investigated regime. Figures 2(b) and 2(c) show the computed counterpart of Fig. 2(a) in the absence and in the presence of dispersive propagation in the imaging optics, respectively. The latter confirms the optimum agreement with the experiment, both in the overall time-wavelength profile and in the value of the chirp of the redshifted continuum (see the full line, with slope $-0.24 \mathrm{~nm} / \mathrm{fs}$ ). Figure 2(b), however, indicates that at the sample output the redshifted components should be actually given by two temporally separated events (indicated with "1" and "2" in the figure), associated to two fronts, the first one with duration of $\sim 55 \mathrm{fs}$ and chirp $-0.7 \mathrm{~nm} / \mathrm{fs}$ and the second one with duration of $\sim 30 \mathrm{fs}$ and chirp $-1.31 \mathrm{~nm} / \mathrm{fs}$. Note that the presence of two redshifted fronts at different delays could be also inferred by the two-spot interference structure featuring the large-delay axial spectra in Figs. 1(b) $-1(\mathrm{~g})$. The fact that the front rise time in Fig. 2(b) is smaller with respect to that shown in Fig. 2(c) shows the relevant impact of dispersive broadening in the imaging optics. The fact that the same quantity is larger than $T_{0}$ shows the importance of the dispersive broadening inside the nonlinear sample, shocks being eventually formed for $z<3 \mathrm{~cm}$.

The analysis of the computed propagation inside the nonlinear sample illustrates how the WP undergoes successive pulse splitting, accompanied by $X$-wave formation and selfsteepening of both trailing and leading fronts. In Fig. 3 the numerically calculated on-axis intensity profiles are shown for different propagation distances. At $z=1.2 \mathrm{~cm}$, a falling shock front is formed on the trailing split pulse, characterized by a HWHM duration of 7 fs. Note the occurrence of fast oscillations at larger delays, which should be taken as signature of the wave breaking usually associated to the formation of a shock front [3]. Similarly, at $z=1.8 \mathrm{~cm}$ a rising shock front develops in the leading split pulse, with a similar HWHM duration of $8.5 \mathrm{fs}$ and an analogous wave-breaking effect. Finally, for $z=1.9 \mathrm{~cm}$ and $z=2.5 \mathrm{~cm}$ two other shocks form in the trailing and leading parts of the WP, respectively. The dynamics between $z=0$ and $z=3 \mathrm{~cm}$ leads therefore to the formation of two falling (indicated with "1" and " 3 ") and two rising (indicated with "2" and "4" in Fig. 3) shock fronts, each of them leading to a distinct on-axis spectral continuum emission. Notably, it is the interference between

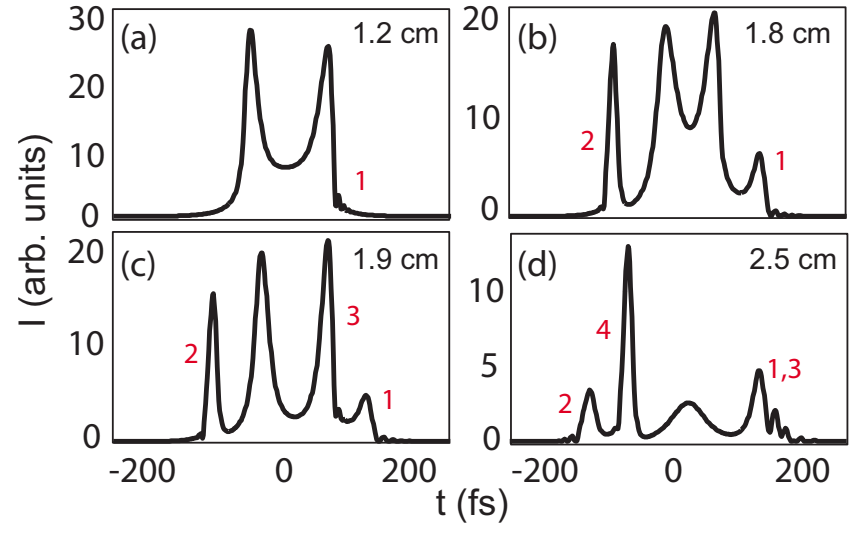

FIG. 3. (Color online) Calculated on-axis intensity profile at propagation distances at which shock events occur.

these temporally shifted shocks that gives rise to the fringes in the axial emission apparent in the time-integrated spectra in Fig. 1(a) [10].

Relying upon far-field AS characterizations, the pulse splitting has been recently described in terms of spontaneous formation of superluminal and subluminal $X$ waves $[12,19]$. Here we draw attention to the near-field profile of the formed $X$ waves, which differs substantially from the symmetrical, biconical (i.e., clepsydralike) shape featuring the exact nonlinear stationary regime [13]. Figure 4(a) shows the entire WP $r$ - $t$ intensity distribution computed for $z=1.8 \mathrm{~cm}$, i.e., at the position in the cuvette where the first leading shock occurs [see the corresponding on-axis profile in Fig. 3(b)]. The inset zoom around the shock shows the actual shape of what we call a "shocked $X$ wave," which travels at superluminal velocity and is composed by a virtually plane-wave steep front on the outer side and a conical structure facing toward the inner portion of the pulse. Figure 4(b) shows the AS corresponding to a $20 \mathrm{fs}$ time slice centered at $\tau=-100 \mathrm{fs}$, i.e., at the leading shock front. Note the extended, redshifted, axial tail, which is free from angular dispersion and corresponds to the plane shock; note also the conical structure at the blueshifted side of the spectrum, where angular disper-
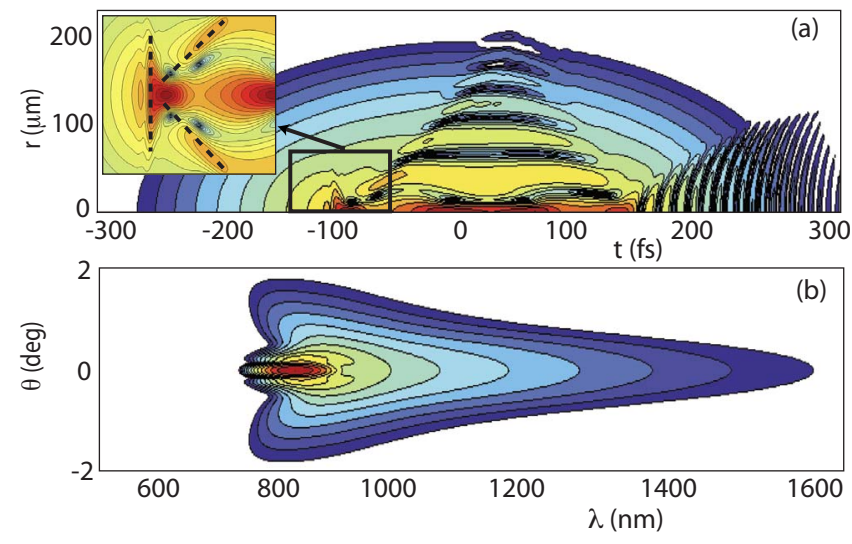

FIG. 4. (Color online) (a) Numerically calculated $r-t$ profile whose enlargement around $t=-100 \mathrm{fs}$ is shown in the inset. (b) Angular spectrum of a $20 \mathrm{fs}$ time slice centered at $t=-103 \mathrm{fs}$. All results are calculated for $z=1.8 \mathrm{~cm}$. 
sion is evident. This result demonstrates that axial and conical emission do not originate from separate temporal portions of the pulse, as one might erroneously infer from data shown in Fig. 1 due to limitation imposed by dispersion. Similar dynamics were observed for the other three shock fronts.

The connection between the axial and conical spectral broadening leads to an original interpretation of the physics lying behind the rising shock-front formation. Indeed, conical emission is an indication of the formation of $X$ waves and, in particular, the pulse splitting process has been interpreted as the formation of two split nonlinear $X$ waves, one traveling with subluminal, the other with superluminal group velocity with respect to the unperturbed background that is traveling at the luminal group velocity $v_{g}^{L}=d \omega /\left.d k\right|_{\omega_{0}}[19]$. In other words, the nonlinearity induces an angular dispersion that in turn induces a variation of the pulse velocity. If we consider the trailing pulse $\left(v_{g}<v_{g}^{L}\right)$ then we recover the well known situation in which the intensity peak travels slower than the surrounding wings and accumulates at the trailing edge. However, for the leading split pulse $\left(v_{g}>v_{g}^{L}\right)$ the opposite will occur and energy will accumulate at the leading edge, thus explaining the formation of the rising shock front.

In conclusion, we have characterized for the first time the dynamics of conical emission (CE) and shock-front forma- tion, generated by filamentation in water, by their timeresolved angular spectra. Our results identify the spatiotemporal localization of the various spectral components of supercontinuum in the $X$ wave, showing that the temporal edges (i.e., rising and falling fronts, respectively, of the leading and trailing pulses) of the WP generate the redshifted and blueshifted axial radiation. This radiation is enhanced by self-steepening and shock-front formation that arises due to the interplay between the Kerr nonlinearity and the subluminal or superluminal group velocity propagation of the intensity peaks. Moreover, the simultaneous presence of $\mathrm{CE}$ and white-light axial supercontinuum in the AS shows that these features are both manifestations of the same physical process, i.e., the generation of what we may call shocked $X$ waves.

The authors gratefully acknowledge the financial support from the Italian Ministry of University and Research (Grants No. COFIN04 and No. FIRB RBIN04NYLH) and from the Access to Research Infrastructures activity in the Sixth Framework Programme of the EU (Contract No. RII3-CT2003-506350, Laserlab Europe). P.D.T. acknowledges the EU Marie Curie Chair action (STELLA), contract MEXC2005-025710.
[1] F. DeMartini, C. H. Townes, T. K. Gustafson, and P. L. Kelley, Phys. Rev. 164, 312 (1967).

[2] R. Z. Sagdeev, in Shock Waves in Plasmas, Reviews of Plasma Physics, Vol. 4, edited by M. A. Leontovich (Consultants Bureau, New York, 1966).

[3] J. E. Rothenberg and D. Grischkowsky, Phys. Rev. Lett. 62, 531 (1989).

[4] A. L. Gaeta, Phys. Rev. Lett. 84, 3582 (2000).

[5] N. Aközbek, M. Scalora, C. M. Bowden, and S. L. Chin, Opt. Commun. 191, 353 (2001).

[6] I. S. Golubtsov and O. G. Kosareva, J. Opt. Technol. 69, 462 (2002).

[7] V. P. Kandidov, O. G. Kosareva, I. S. Golubtsov, W. Liu, A. Becker, N. Aközbek, C. M. Bowden, and S. L. Chin, Appl. Phys. B: Lasers Opt. 77, 149 (2003).

[8] A. A. Zozulya, S. A. Diddams, A. G. Van Engen, and T. S. Clement, Phys. Rev. Lett. 82, 1430 (1999).

[9] A. Couairon and A. Mysyrowicz, Phys. Rep. 441, 47 (2007).

[10] D. Faccio, P. Di Trapani, S. Minardi, A. Bramati, F. Bragheri, C. Liberale, V. Degiorgio, A. Dubietis, and A. Matijosius, J. Opt. Soc. Am. B 22, 862 (2005).
[11] A. Couairon, E. Gaizauskas, D. Faccio, A. Dubietis, and P. Di Trapani, Phys. Rev. E 73, 016608 (2006).

[12] M. Kolesik, E. M. Wright, and J. V. Moloney, Phys. Rev. Lett. 92, 253901 (2004).

[13] C. Conti, S. Trillo, P. Di Trapani, G. Valiulis, A. Piskarskas, O. Jedrkiewicz, and J. Trull, Phys. Rev. Lett. 90, 170406 (2003).

[14] M. Kolesik, E. M. Wright, and J. V. Moloney, Opt. Express 13, 10729 (2005).

[15] F. Bragheri, C. Liberale, V. Degiorgio, D. Faccio, A. Matijosius, G. Tamosauskas, A. Varanavicius, and P. Di Trapani, Opt. Commun. 256, 166 (2005).

[16] M. Kolesik, G. Katona, J. V. Moloney, and E. M. Wright, Phys. Rev. Lett. 91, 043905 (2003).

[17] D. Faccio, A. Matijosius, A. Dubietis, R. Piskarskas, A. Varanavicius, E. Gaizauskas, A. Piskarskas A. Couairon, and P. Di Trapani, Phys. Rev. E 72, 037601 (2005).

[18] A. A. Zozulya, S. A. Diddams, and T. S. Clement, Phys. Rev. A 58, 3303 (1998).

[19] D. Faccio, M. A. Porras, A. Dubietis, F. Bragheri, A. Couairon, and P. Di Trapani, Phys. Rev. Lett. 96, 193901 (2006). 